\title{
The size of an attentional window affects working memory guidance
}

\author{
Mireia Hernández \\ University of Barcelona, Barcelona, Spain \\ Albert Costa \\ Catalan Institution for Research and Advanced Studies, Barcelona, Spain \\ and Pompeu Fabra University, Barcelona, Spain \\ AND \\ GLYN W. HuMPHREYS \\ University of Birmingham, England, Birmingham
}

\begin{abstract}
We ask whether attentional guidance from working memory (WM) is influenced by the size of an attentional window. Participants adopted either a focused or a diffuse attentional window when responding to a search display. Prior to the search display an initial cue had to be held in memory (Experiment 1A, visual WM; Experiment 1C, verbal WM) or merely identified (Experiment 1B, identification). In all cases, search performance was affected by the re-presentation of the cue in the search display, with the cuing effects (either cost or benefit) being larger when the cue was held in memory than when it was merely identified. Critically, the magnitude of the cuing benefit increased when participants adopted a diffuse attentional window. This held for effects that are based on items held in WM and for effects that are based only on item priming. The results suggest that variations in the size of an attentional window modulate top-down (both WM conditions) as well as bottom-up guidance of attention (identification condition).
\end{abstract}

There is considerable evidence that visual attention can be guided by irrelevant distractors. Effects of bottom-up attentional guidance have been demonstrated by studies showing that attention can be drawn to a distractor singleton instead of to a target in a search display (e.g., Theeuwes, 1992). Effects of top-down attentional guidance have been apparent in experiments showing that attention can be directed to a distractor that matches task-irrelevant information held in working memory (WM; e.g., Downing, 2000; Soto, Heinke, Humphreys, \& Blanco, 2005). The effects of distractors that are cued from WM can occur even when the stimuli in WM are always invalid and never cue a target (Soto et al., 2005), demonstrating that it is difficult to prevent distraction when new stimuli match items in WM.

Studies of the bottom-up capture of attention have shown that some control over attentional guidance can be brought about by observers varying the size of the attentional window that they adopt. When spatial attention is focused-for example, using a narrow (focused) attentional window-the effects of salient distractors may be reduced relative to when a wider (diffuse) attentional window is adopted (see, e.g., Theeuwes, 2004; see also Gibson and Peterson, 2001; Theeuwes, Kramer, \& Belopolsky, 2004). Recent evidence for this proposal came from Belopolsky, Zwaan, Theeuwes, and Kramer (2007), who examined the effects of bottom-up saliency on visual selection. Participants were required to carry out a visual search task in which they had to detect a shape target with relatively low saliency in the presence of a salient singleton color. Belopolsky et al. manipulated the size of an attentional window by requiring different tasks to be performed prior to initiating search for the target. In the focused attentional window, observers were instructed to start searching for the target after checking a central fixation point. In the diffuse attentional window, observers started searching after checking the global shape formed by the elements making up the search array. Reaction times (RTs) were faster overall in the diffuse attentional window than in the focused attentional window, consistent with a greater degree of parallel processing in the diffuse attentional window. In addition, an irrelevant singleton affected only target search when participants adopted a diffuse attentional window (see also Yantis \& Jonides, 1990, for earlier evidence). Apparently, the effects of bottomup attentional guidance can be filtered out when participants adopt a narrow, focused attentional window. On the other hand, bottom-up saliency overruled the effects of task-based control of selection once stimuli fell within a more distributed attentional window. Belopolsky et al.

G.W. Humphreys, g.w.humphreys@bham.ac.uk 
speculated that there was no top-down control of selection other than manipulations of the attentional window, and that within an attended area selection was driven in a purely bottom-up manner.

In the present study, we asked whether variations in the size of an attentional window modulate not only effects of bottom-up capture of attention but also effects of topdown guidance that are induced by stimuli in a search display matching task-irrelevant information held in WM. In doing this, we tested whether there is top-down guidance of attention within a given attentional window. Note that the notion of top-down attentional guidance that is used in the present article differs from the one in Belopolsky et al. (2007). Belopolsky et al. used the term top-down attentional guidance to refer to any strategy voluntarily developed by participants in order to avoid being captured by irrelevant physical properties of the items. In the present article, however, we use the term top-down attentional guidance to refer to the case in which attention is guided by intrinsic information (i.e., items held in WM) in contrast to attention being guided, bottom-up, by extrinsic information in the displays (e.g., the physical properties of the items).

To manipulate top-down guidance, we used search displays in which one stimulus could sometimes match an irrelevant representation held in WM: Prior evidence indicates that this matching item has a strong influence on attentional guidance, influencing the first eye movement made during search (Soto et al., 2005) and altering perceptual sensitivity to targets (Soto \& Humphreys, 2009). Here, we ask whether this top-down modulation of search is dependent on whether participants adopt a focused or a diffuse attentional window. Let us imagine that top-down matching can be applied to only one item at a time. If this were the case, then the effects would be at least as strong when a focused attentional window is adopted (where one item is considered at a time) as when a diffuse window is employed. If a representation in WM is matched against display items in parallel, however, then the effects of the WM stimulus might be stronger when a diffuse attentional window is employed (enabling parallel matching to operate) than when attention is more focused. We report data from three experiments in which we manipulated the size of an attentional window prior to search in a manner similar to that used in Belopolsky et al. (2007). In Experiment 1A (the visual WM condition), participants had to hold a colored form conjunction in memory prior to searching for an oriented line target (cf. Soto et al., 2005). The item in memory could reappear in the search display, where it could contain either the target (on valid trials) or a distractor line (on invalid trials). Following search, memory for the initial conjunction stimulus was assessed. We tested for effects of cue validity on target search. In Experiment 1B (the repetition condition), we presented identical displays up to the search task, but participants had only to identify the first conjunction rather than to hold it in memory. Effects of bottom-up priming of the perceptual system from the initial cue were matched in the experiments, and participants were forced to hold the stimuli in WM only in Experiment 1A. If there are effects of WM over and above bottom-up perceptual priming, then cue validity should have had a stronger influence in Experiment 1A than in Experiment 1B. A further test of top-down factors is reported in Experiment 1C, in which we used a verbal WM cue rather than a visual cue (the verbal WM condition), with memory for the verbal cue again being tested after the search display. The verbal cue eliminated effects of bottom-up perceptual priming so that any effects of the verbal cue on subsequent search would have to emerge in a top-down manner. We asked whether these top-down effects are contingent on participants' adopting a diffuse attentional window.

\section{METHOD}

\section{Participants}

Ninety-two volunteers (16 men, 76 women) took part in this study. Thirty participants took part in Experiment 1A (visual WM), 31 in Experiment 1B (identification), and 31 in Experiment 1C (verbal WM). Originally, 33 participants were included in each experiment, but 3 participants in Experiment $1 \mathrm{~A}$ and 2 participants in each of Experiments 1B and 1C were excluded because of their high error rate in the no-go condition ( $>35 \%$; see below). Fifty-two participants were undergraduate students from the University of Birmingham, and 40 were undergraduate students from the University of Barcelona. The participants ranged in age from 19 to 28 years, and all had normal or corrected-to-normal vision and normal color vision.

\section{Design and Procedure}

The task was always to search for the only tilted line present in the display. Participants were instructed to press the "M" key if the line was tilted toward the right and "V" if it was tilted toward the left. The search display consisted of eight colored line figures presented against a black background. Together, the figures formed the global shape of an equilateral triangle that (randomly) pointed either up or down. This global shape could have "E" or "F" presented in its center. The shapes marking up the global form were outline circles, diamonds, squares, crosses, and hexagons. The color of the figures could be red, green, blue, yellow, or pink. There were always eight exemplars making up the global figure. The same conjunction of color and shape never appeared more than once in a search array, although there were always two instances of each shape and two of each color. Each figure in the search display contained a white line. All of the lines were vertical except for one - the target - which was tilted $26^{\circ}$ to either the left or the right. The size of each shape was $1.80^{\circ} \times 1.80^{\circ}$ for the circle, $1.91^{\circ} \times 1.91^{\circ}$ for the diamond, $1.50^{\circ} \times 1.50^{\circ}$ for the square, $2.0^{\circ} \times 1.5^{\circ}$ for the cross, and $2.38^{\circ} \times$ $0.95^{\circ}$ for the hexagon. The length of the line was $0.57^{\circ}$, and its width was $0.12^{\circ}$

There were two attentional conditions within each experiment. In the focused attention condition, participants had to start searching only when the fixation point was an E (go trials), and they had to withhold their responses when the fixation point was an $\mathrm{F}$ (no-go trials). In the diffuse attention condition, participants had to start searching only when the global triangle shape was pointing upward (go trials), and they had to withhold their responses when the global shape pointed downward (no-go trials) (see Figure 1). The no-go signal occurred on $25 \%$ of all trials. The same displays were used for both the focused and the diffuse attention conditions, except for the no-go signal (either the $\mathrm{F}$ at fixation or an inverted triangle). The two attention conditions were presented in separate blocks. Half of the participants performed under the focused attention condition first, and the other half performed under the diffuse 


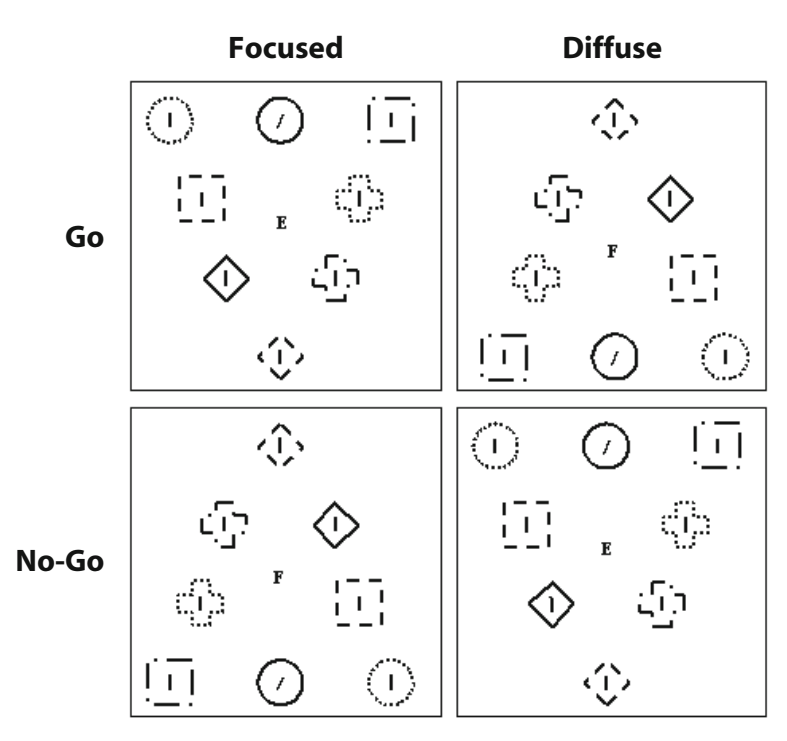

Figure 1. Examples of the displays used to manipulate the size
of the attentional window (focused, diffuse). In the focused at-
tentional window condition, participants had to start searching
for the tilted line only when the fixation point was an E. In the
diffuse attentional window condition, participants had to start
searching for the tilted line only when the global shape was an
upward-pointing triangle.

attention condition first. There were 90 trials in each block ( 72 go, 18 no-go).

Before the start of each block, the participants received a sample block of 24 practice trials with the same proportion of cue validity and of go and no-go trials as the experimental blocks. Stimuli were presented in DMDX (Forster \& Forster, 2003).

There were three experiments in which we varied the task performed on a cue that was given prior to the search display:

Experiment 1A. In Experiment 1A (visual WM), a visual cue (a color-shape conjunction) was initially presented. Participants were instructed to keep the conjunction in memory while responding as quickly as possible to the orientation of the tilted-line target in the search display. Immediately after the presentation of the search display, a memory probe appeared in order to make sure that participants kept the cue in memory. On these probe trials, a figure was displayed below the question "Different or Same?" Participants had to indicate whether this figure was identical (both in color and in shape) to the cue or not by pressing "V" for different and "M" for same. On different trials, the probe had a shape or color contrasting with the initial cue, ensuring that the conjunction had to be maintained. Participants were asked to perform well on both the visual search and the memory-probe tasks.

The initial cue was a circle, a diamond, a square, a cross, or a hexagon, and its color was red, green, blue, yellow, or pink. The cue was either present in or absent from the search array and, when present, contained either the target or a distractor. This led to three types of trial, which appeared randomly. On valid trials, the target appeared within the re-presented cue. On invalid trials, the target appeared in a shape that was different from that of the re-presented cue. On neutral trials, the memory cue was not re-presented in the search array, and no other figure in the array had the same combination of color and shape. On all trial types, at least one item in the display matched the color, and another item matched the shape of the memory cue, so the re-presentation conditions here (on valid and invalid trials) involved a repeat of the complete memorized conjunction of color and shape. There were 24 trials for each condition of cue validity (invalid, valid, neutral) in each of the two attention blocks (focused, diffuse). Each cue appeared once for each cue-validity condition.

Each trial began with the presentation of a fixation point for $500 \mathrm{msec}$. Then, two instances of a cue (the memory item) were flashed at fixation for brief presentations of $100 \mathrm{msec}$ and $500 \mathrm{msec}$, separated by a blank interval of $100 \mathrm{msec}$. At $250 \mathrm{msec}$ after the cue disappeared, the search array was presented and remained visible until a response was given (or until 3,000 msec passed). After participants responded to the orientation of the tilted line, a memory probe appeared for $3,000 \mathrm{msec}$, or until a response was given (see Figure 2A). The interval between the cue and the search display was slightly longer than that used in some previous experiments (e.g., Soto et al., 2005) but not longer than that used in others (Soto \& Humphreys, 2007; see also the present Experiment 1C). The previous results showed that performance was little affected by this interval, suggesting that the WM effects are not confined to those instances in which stimuli are being encoded.

Experiment 1B. In Experiment 1B (identification), the design and procedure up to the appearance of the search display were identical to those in Experiment 1A (visual WM), except that observers were not required to keep the cue in memory.

Instead, participants were instructed to compare the two instances of the cue. If the instances matched, participants carried out the search task (as in Experiment 1A).

When the two instances differed (in either color or shape; see Figure 2B), participants had to withhold their response to the search display. Trials in which the two instances of the cue did not match served as catch trials and they occurred on $20 \%$ of the trials, in each block (with focused attention and with diffuse attention). There were 15 catch trials (and 57 repetition trials) out of 72 go trials. The proportions of catch and repetition trials were equally distributed across the three levels of cue validity (invalid, neutral, valid). There were thus 5 catch trials and 19 repetition trials for each level of cue validity within a block. This identification condition was an adaptation of the baseline that was used in Soto et al. (2005, Experiment 3) and in Soto, Humphreys, and Rotshtein (2007).

Experiment 1C. Experiment 1C (verbal WM) replicated Experiment $1 \mathrm{~A}$, except that a verbal rather than a visual WM task was employed. Here, the cue was a written phrase specifying both the color and shape of a figure (e.g., a red circle). Participants were instructed to keep this verbal description in memory, and they were tested for the conjunction of properties with a verbal probe that was presented after the search task (e.g., same probe = red circle, different probe $=$ red square or blue circle). On valid and invalid trials, the verbal cue described the conjunction that was represented by one of the stimuli in the search display; on neutral trials, a matching conjunction was not present. In all cases, however, one other item in the search display had the color, and another item had the shape, corresponding to the name of the color or the shape given by the cue. As in Experiments $1 \mathrm{~A}$ and 1B, the cue was valid when the re-presented item contained the target, and it was invalid when it contained a distractor.

Each trial began with a fixation point lasting $500 \mathrm{msec}$. After this, a verbal cue (the memory item) was presented for $2,000 \mathrm{msec}$. The cue was presented for longer here than in Experiments $1 \mathrm{~A}$ and $1 \mathrm{~B}$ to ensure that the words were read correctly. Note, however, that this duration matches that used by Soto and Humphreys (2007) when they successfully showed that irrelevant verbal cues held in WM could have an inadvertent effect on a subsequent search task. At $250 \mathrm{msec}$ after the cue disappeared, the search array was presented and stayed visible until either a response was given or 3,000 msec had passed. After the participants had responded to the orientation of the tilted line, a verbal memory probe was presented for $3,000 \mathrm{msec}$, or until a response was given (see Figure 2C). 


\section{A}

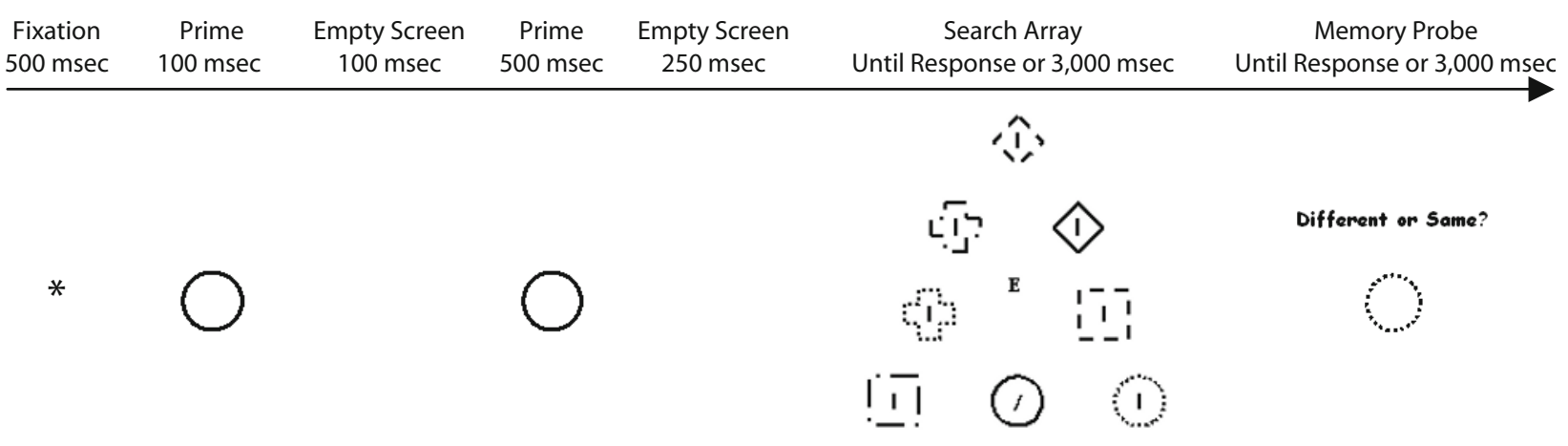

B

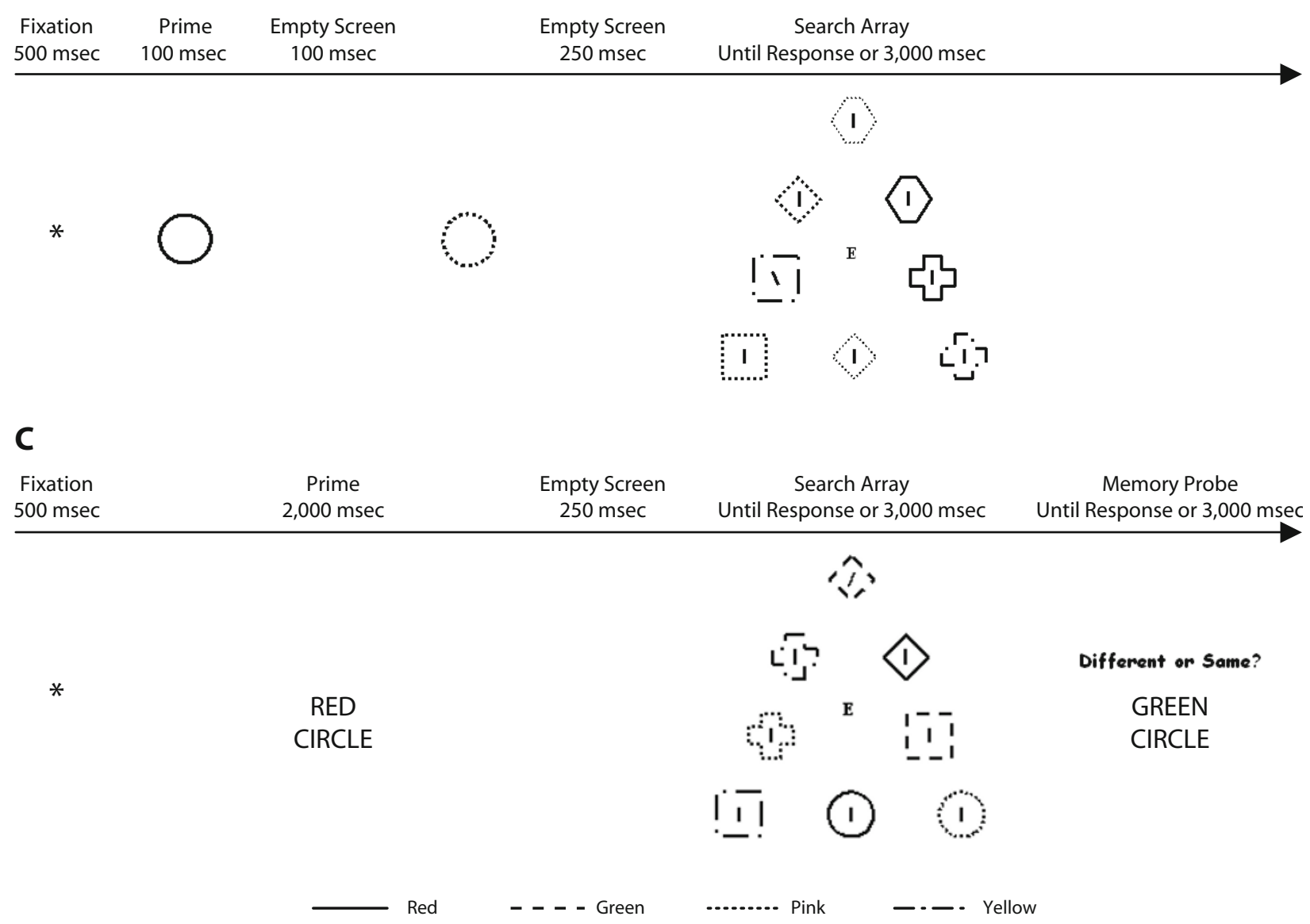

Figure 2. Display sequences for each experiment. (A) An example of the display representing a valid trial in Experiment 1A (visual working memory). (B) An example of the display representing an invalid trial in Experiment 1B (identification). (C) An example of the display representing a neutral trial in Experiment 1C (verbal working memory). Participants should have responded "different" to the memory probe of the three examples.

\section{RESULTS}

Trials with errors were excluded from the analysis, as well as with no-go trials and trials with RTs that were greater than $2.5 S D$ s from the mean. This last action led to the loss of fewer than $1.5 \%$ of the trials in Experiment $1 \mathrm{~A}$ (visual WM), fewer than $2.1 \%$ of the trials in Experi- ment 1B (identification), and fewer than $2 \%$ of the trials in Experiment $1 \mathrm{C}$ (verbal WM).

We first conducted a general ANOVA on correct RTs with attentional window (focused, diffuse) and cue validity (invalid, neutral, valid) as within-subjects factors and experiment (visual WM, identification, verbal WM) as a between-subjects factor. 
There was a reliable main effect of experiment $\left[F(1,89)=5.38, M S_{\mathrm{e}}=311,593.93, p<.006\right]$. Planned comparisons showed slower performance in the visual WM conditions $(1,412 \mathrm{msec})$ than in the identification $(1,223 \mathrm{msec})$ and verbal WM $(1,293 \mathrm{msec})$ (both $p \mathrm{~s}<.04)$ conditions; there was no significant difference between the identification and verbal WM conditions. A main effect of attentional window $\left[F(1,89)=20.82, M S_{\mathrm{e}}=20,807.75\right.$, $p<.0001]$ was observed, which reflected longer RTs when there was a focused $(1,337 \mathrm{msec})$ versus a diffuse $(1,281 \mathrm{msec})$ attentional window. The main effect of cue validity was also significant $\left[F(2,178)=124.75, M S_{\mathrm{e}}=\right.$ $13,051.45, p<.0001]$. Planned comparisons showed slower performance for invalid trials $(1,401 \mathrm{msec})$ than for neutral trials $(1,314 \mathrm{msec})$, and faster performance in the valid trials $(1,213 \mathrm{msec})$ than in the neutral trials (all $p \mathrm{~s}<.0001)$; thus, there were both benefits and costs from the cue, and these effects were equivalent in magnitude $[t(91)=0.87, p<.34]$.

The two-way interaction between cue validity and experiment was also significant $\left[F(4,178)=28.37, M S_{\mathrm{e}}=\right.$ $13,051.45, p<.0001]$, indicating that the magnitude of the cuing effect varied across the experiments. Both the costs and the benefits of the cue were larger in Experiment 1A (visual WM) than in Experiment 1B (identification) (both $p \mathrm{~s}<.001$; benefits were $176 \mathrm{msec}$ larger and costs were $120 \mathrm{msec}$ greater in Experiment 1A). There were also greater costs and benefits in Experiment 1A (visual WM) than in Experiment 1C (verbal WM) (all $p$ s $<$ .002 ; benefits were $148 \mathrm{msec}$ greater and costs $80 \mathrm{msec}$ greater in Experiment 1A than Experiment 1C). The costs were also greater in Experiment 1C (verbal WM) than in Experiment 1B (identification) (a difference of $40 \mathrm{msec}$, $p<.04$ ).

Crucially, for our purposes, the two-way interaction between attentional window and cue validity was significant $\left[F(2,178)=4.39, M S_{\mathrm{e}}=5,620.19, p<.01\right]$. This was examined by comparing RTs in the focused attention condition with those in the diffuse attention condition for each cue-validity condition. For all cue-validity conditions, RTs were slower in the focused attention condition than in the diffuse attention condition (an effect size of $41 \mathrm{msec}$ for invalid cues, $44 \mathrm{msec}$ for neutral cues, and $82 \mathrm{msec}$ for valid cues; see Figure 3). The beneficial effect of the WM cue (on valid trials relative to neutral trials) was $38 \mathrm{msec}$ larger in the diffuse attention condition than in the focused attention condition $[t(91)=$ $2.63, p=.01]$ (see Figure 4). In contrast, the cost effects of the WM cue on invalid trials did not vary as a function of the attention condition $[t(91)<0.19, p<.85]$. The lack of the attentional window $\times$ cue validity $\times$ experiment three-way interaction $[F(4,178)<1]$ indicated that performances in the three experiments (visual WM, identification, and verbal WM) were modulated equally by the attentional window that was adopted (focused or diffuse).

Overall, participants had an error rate of $9.79 \%$ on the no-go trials $(8.63 \%$ in the focused attentional window, $10.95 \%$ in the diffuse attentional window) and an error rate of $2.48 \%$ on the go trials. Performance on memoryprobe trials was high ( $95 \%$ correct cue recognition) and showed no effect of validity. All the error data are reported in the Appendix, including an analysis assessing the effects of cue validity on memory performance.

\section{DISCUSSION}

We found that there were beneficial effects of cuing when the cue was represented in the search display and coincided with a target, and that there were costs to search when the cue reappeared but contained a distractor. These effects were strongest in the visual WM condition, followed by the verbal WM condition and, finally, the identification condition. The fact that the cuing effects were larger in the two WM conditions than in the identification condition indicates that there was a substantial top-down component to the effect, and that performance was not driven simply by bottom-up priming of the perceptual system following the presentation of the cue. Indeed, it is difficult to know whether the effects that occurred in the identification condition were themselves caused by participants sometimes holding the first stimulus in WM rather than by mere perceptual priming from the cue; however, the verbal WM condition showed that the effects emerged even when any bottom-up priming was eliminated. These results replicate data reported by Downing (2000), Soto and Humphreys (2007), and Soto et al. (2005).

On top of this finding, we showed that the effects of cue validity were modulated by our manipulation of the size of the attentional window. Overall, RTs were faster in the diffuse attention condition than in the focused attention condition, which is consistent with a greater degree of parallel processing of the displays in the diffuse condition (see also Belopolsky et al., 2007). In addition, the cue-validity effects were greater under diffuse attention conditions (though effects were still present in the focused attention condition). This modulation of the cuing effect indicates that the size of an attentional window can influence the degree to which information held in WM can influence attention in a top-down manner. The results are consistent with the proposal that the representation in WM is matched against members of the search display in a spatially parallel manner, rather than matching being confined to one item at a time. This parallel matching process can operate more effectively when spatial attention is distributed across the whole display. The argument that a memory template can be matched in parallel across members of a search display supports other recent data showing advertent effects on search of presenting a distractor that is semantically related to a target (e.g., presenting a motorbike when the task is to search for a motorbike helmet; Belke, Humphreys, Watson, Meyer, \& Telling, 2008; Chelazzi, Miller, Duncan, \& Desimone, 1993). Belke et al. reported that this semantic effect on search was additive across different display sizes, suggesting that semantic information about display members is matched against the memory item in parallel across the displays. 

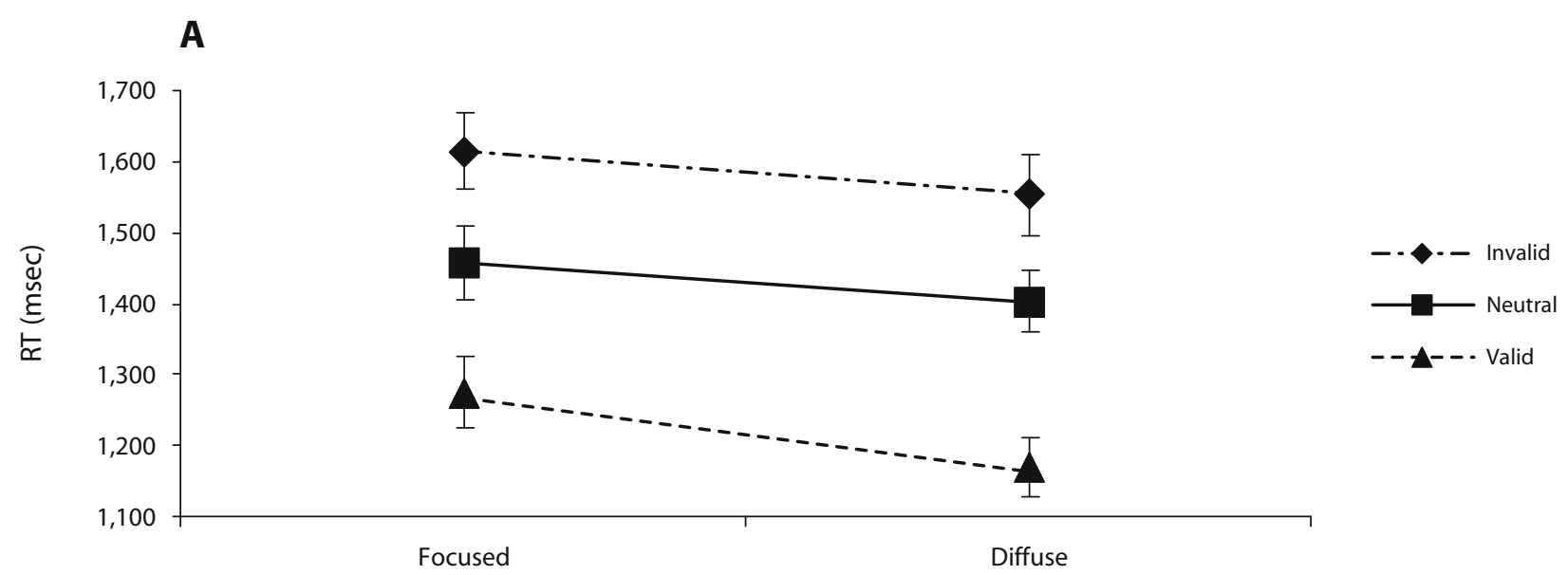

Size of the Attentional Window

B

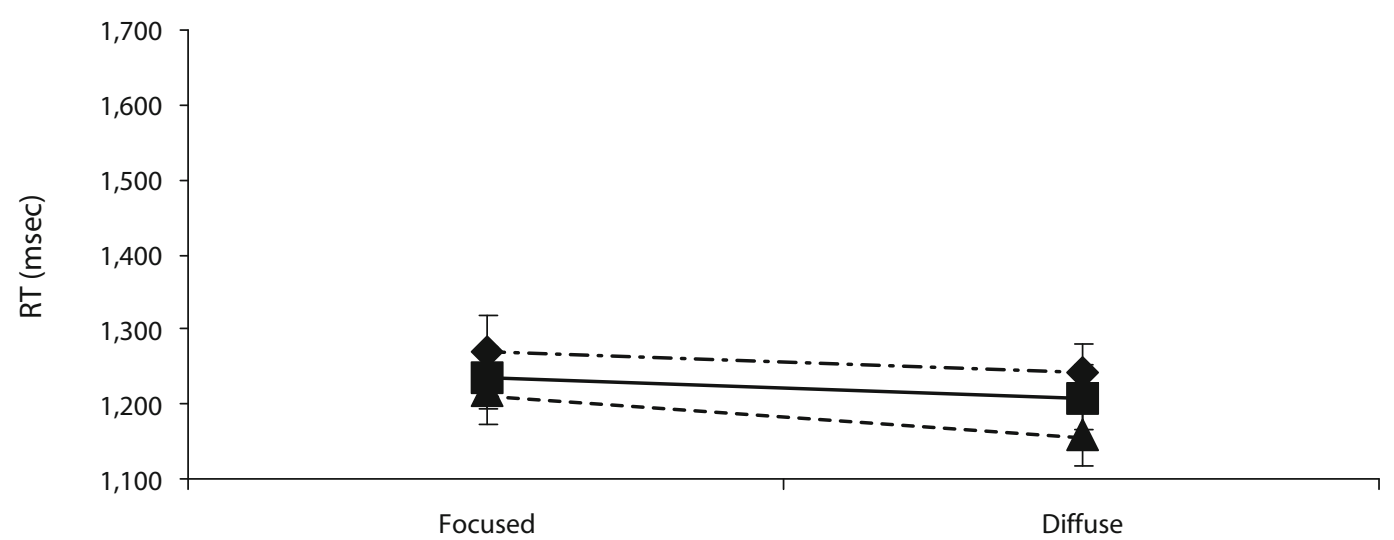

$-\cdot-$ Invalid

$\longrightarrow$ Neutral

- $-\mathbf{A}-\cdot$ Valid

Size of the Attentional Window

C

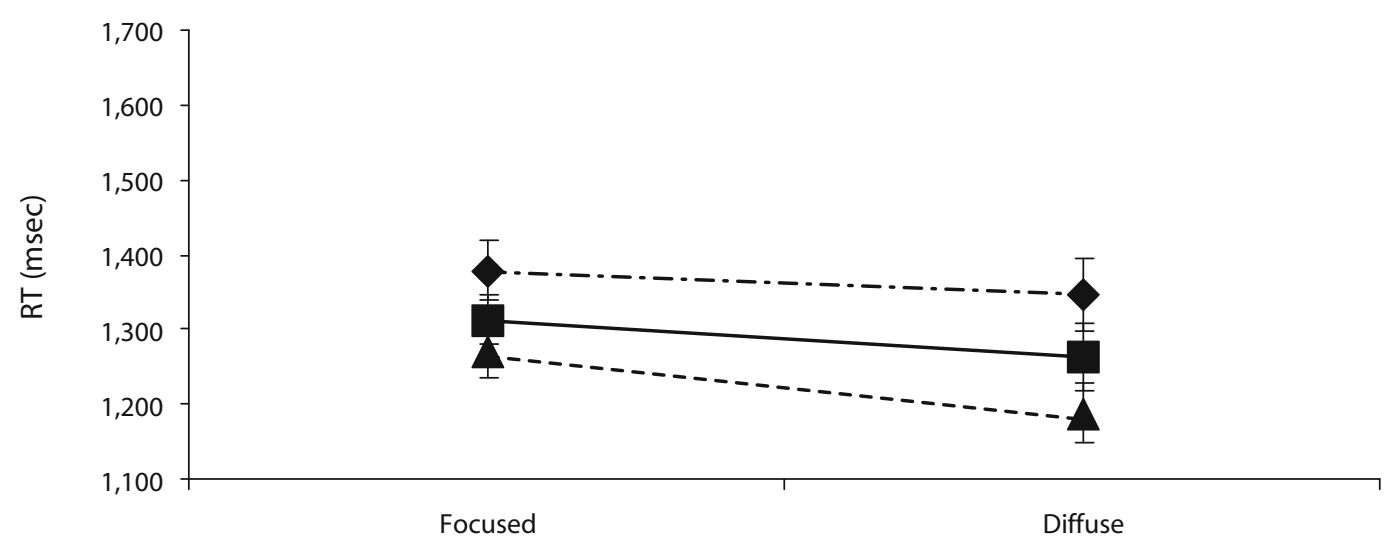

Size of the Attentional Window

Figure 3. (A) Reaction times (RTs) for Experiment $1 \mathrm{~A}$ (visual working memory) as a function of the size of the attentional window (focused, diffuse) and cue validity. (B) RTs for Experiment 1B (identification) as a function of the size of the attentional window (focused, diffuse) and cue validity. (C) RTs for the Experiment 1C (verbal working memory) as a function of the size of the attentional window (focused, diffuse) and cue validity. 
A

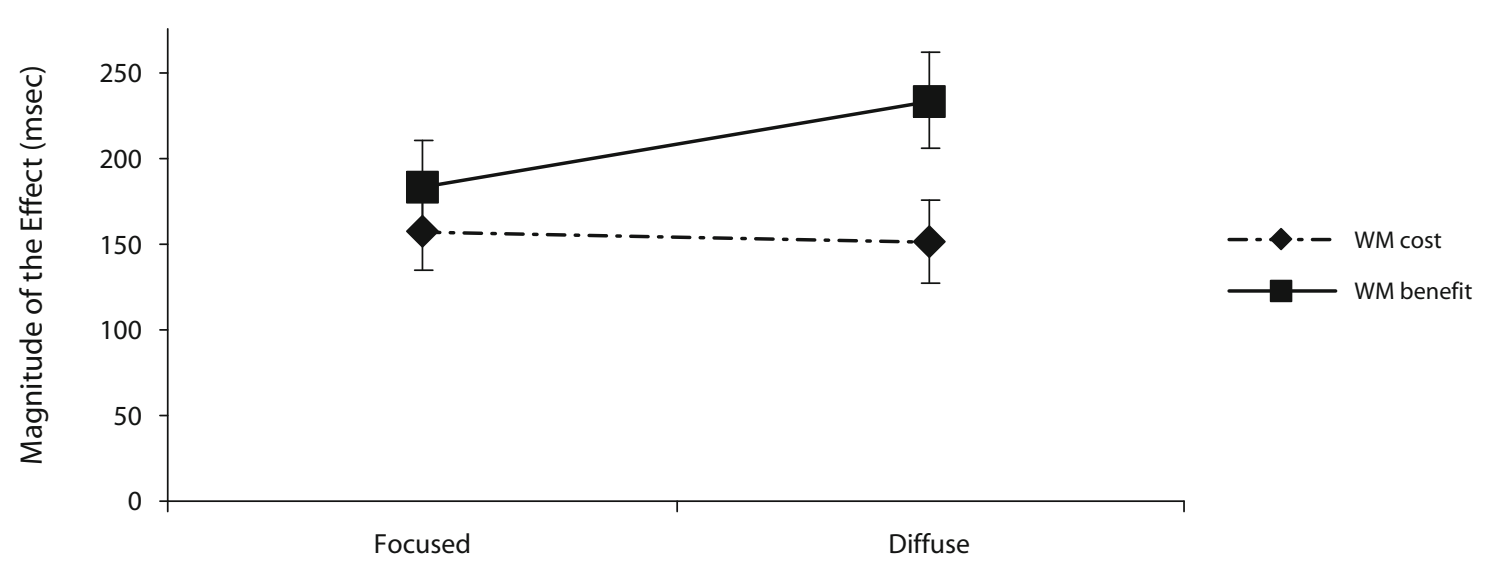

Size of the Attentional Window

B

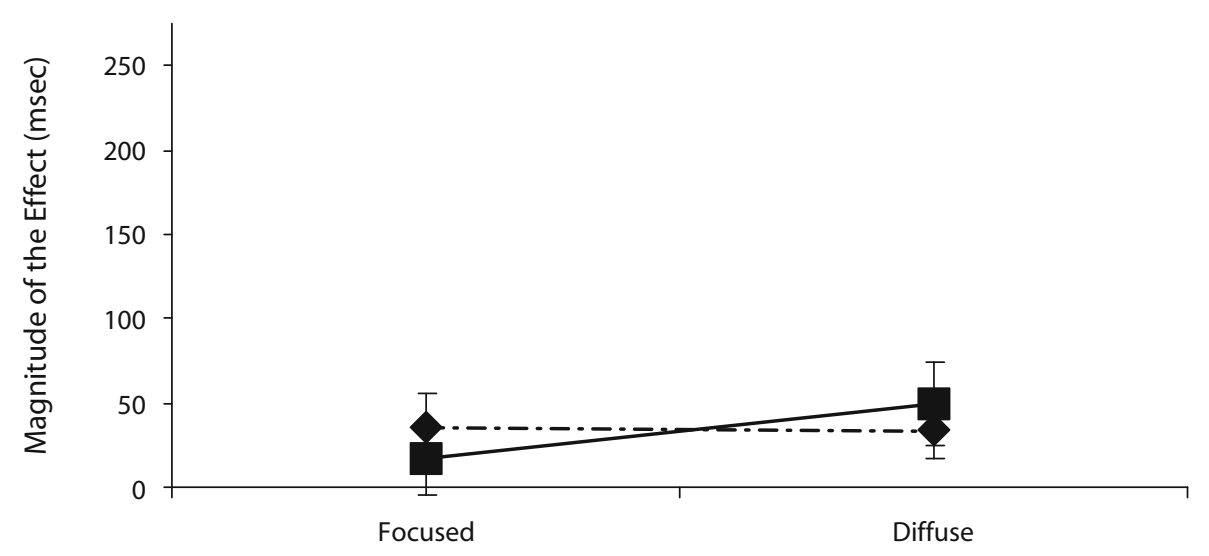

Size of the Attentional Window

C

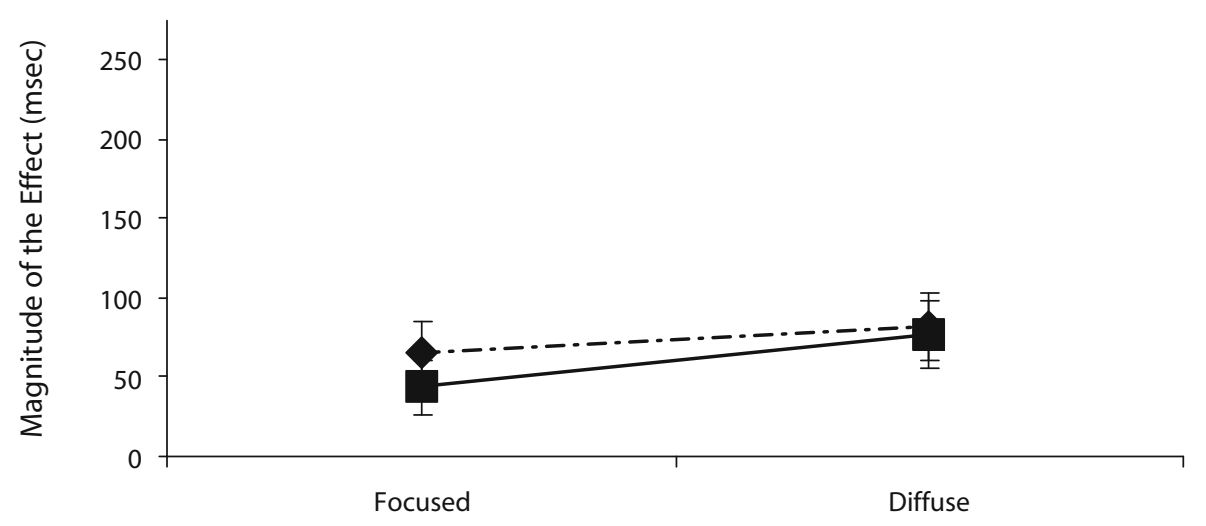

Size of the Attentional Window

Figure 4. (A) Magnitude of the working memory (WM) cost (invalid cues - neutral cues) and WM benefit (neutral cues - valid cues) for Experiment 1A (visual WM) as a function of the size of the attentional window. (B) Magnitude of the WM cost (invalid cues - neutral cues) and WM benefit (neutral cues - valid cues) for Experiment 1B (identification) on the basis of the size of the attentional window. (C) Magnitude of the WM cost (invalid cues - neutral cues) and WM benefit (neutral cues - valid cues) for Experiment $1 \mathrm{C}$ (verbal WM) as a function of the size of the attentional window. 
Once the diffuse attentional window was adopted in the present experiment, furthermore, there was clear evidence for top-down guidance. This is inconsistent with the idea that once the attentional window is set, attentional guidance operates in a purely bottom-up manner (Belopolsky et al., 2007).

One interesting aspect of our data is that the benefit from the cue (on valid trials) was larger in the diffuse attentional window than in the focused attentional window, whereas the cost (on invalid trials) was unaffected by the size of the attentional window. This pattern of results is in line with Belopolsky et al. (2007). In a task with a clear bottom-up component, these authors found that an irrelevant nontarget singleton (relative to a target singleton) captured attention when a diffuse (but not a focused) attentional window was adopted. Note, however, that Belopolsky et al. did not include neutral trials, which did not allow them to clearly differentiate singleton benefits from costs. More importantly, we found that the effect of the cue was modulated by the size of the attentional window in both the present experiment with a clear top-down component (the verbal WM condition) and that with a clear bottom-up component (the identification condition). This indicates that the size of the attentional window affects not only bottom-up attentional guidance (as suggested by Belopolsky et al., 2007) but also the top-down control of attention.

To account for our results, we suggest that-compared with when there is a narrow attentional window-having a wide attentional window increases the probability that attention will be captured by an item in the display matching a stimulus that is held in WM. This will occur on both valid and invalid trials. On valid trials, this capture of attention facilitates search performance. On invalid trials, this same process disrupts performance, because attention initially goes to a distractor rather than to the search target. If this were the only critical factor, however, we would expect the cost from invalid cuing to increase alongside the benefits from valid cuing in the diffuse attention condition more than it does in the focused attention condition. It did not. To explain this, we suggest that increased costs from the greater likelihood of orienting to the WM stimulus in the diffuse condition were offset by attention being more easily disengaged. The resultant trade-off between an increased likelihood of attending to a distractor and the ease of disengaging attention leads to equal costs from invalid cuing under diffuse and focused attention conditions. This account will hold for effects of bottom-up as well as topdown attentional capture, and so also helps to explain the results reported by Belopolsky et al. (2007).

We make two other final points. One is that there may be an interaction between top-down attentional guidance from WM and the size of an attentional window. In the present study, we have shown that engaging participants in a task that promotes a diffuse attentional state can enhance the effects of top-down attentional guidance. Kim and Humphreys (2010) have recently found that items in WM modulate subsequent search most strongly when they match global rather than local properties of the search display. For example, responses to compound hierarchical forms are affected most when an item in WM matches the global versus the local shape (benefiting performance when the global shape matches an item in WM and disrupting performance when there is a mismatch). The process of matching to memory thus seems to operate most efficiently at a global level of display representation. It may be that this, combined with participants being in a diffuse attentional state, maximizes the effects of guidance from WM. The second point is to highlight that the stimulus in WM is task irrelevant in our paradigm. In line with other authors (e.g., Downing, 2000; Olivers, 2009; Soto et al., 2005), we propose that the tendency to attend to the reappearance of the WM in the display reflects automatic guidance of attention. Some authors (e.g., Woodman \& Luck, 2007) have proposed instead that the top-down guidance effects are strategic; participants attend to the reappearance of the WM stimulus to help refresh representations required for the subsequent memory test. We have examined this by assessing whether memory performance was influenced by cue validity (if there is strategic refreshing of memory, memory should be stronger on both valid and invalid trials than on neutral trials). These results are reported in the Appendix. There was no evidence for a memory advantage for the valid and invalid conditions, even though memory performance was not at ceiling. These data are contrary to the memory-refresh account. There also seem to be few reasons to argue that memory refreshing would be more likely under diffuse versus focused attention conditions. One aspect of being in a diffuse attentional state, however, may be that there is weaker task-based control of attention (in the present case, on the basis of a representation of the target for the search task), which enables irrelevant information in WM to also influence performance. Paradoxically, this reduction in task-based control may increase top-down guidance from WM.

\section{AUTHOR NOTE}

This work was supported by a grant from the Medical Research Council (U.K.) to G.W.H. Correspondence concerning this article should be addressed to G. W. Humphreys, Behavioural Brain Sciences, School of Psychology, University of Birmingham, Edgbaston, Birmingham B15 2TT, England (e-mail: g.w.humphreys@bham.ac.uk).

\section{REFERENCES}

Belke, E., Humphreys, G. W., Watson, D. G., Meyer, A. S., \& TellING, A. L. (2008). Top-down effects of semantic knowledge in visual search are modulated by cognitive but not perceptual load. Perception \& Psychophysics, 70, 1444-1458.

Belopolsky, A. V., Zwann, L., Theeuwes, J., \& Kramer, A. F. (2007) The size of on attentional window modulates attentional capture by color singletons. Psychonomic Bulletin \& Review, 14, 934-938.

Chelazzi, L., Miller, E. K., Duncan, J., \& Desimone, R. (1993). A neural basis for visual search in inferior temporal cortex. Nature, 363, 345-347.

DownING, P. E. (2000). Interactions between visual working memory and selective attention. Psychological Science, 11, 467-473.

Forster, K. I., \& Forster, J. C. (2003). DMDX: A Windows display program with millisecond accuracy. Behavior Research Methods, Instruments, \& Computers, 35, 116-124.

Gibson, B. S., \& Peterson, M. A. (2001). Inattentional blindness and attentional capture: Evidence for attention-based theories of visual salience. In C. L. Folk \& B. S. Gibson (Eds.), Attraction, distraction, 
and action: Multiple perspectives on attentional capture (pp. 51-76). New York: Elsevier.

Kim, J., \& HumphreYs, G. W. (2010). Differentiating the effects of working memory and perceptual priming on the perception of hierarchical forms. Manuscript submitted for publication.

Olivers, C. N. L. (2009). What drives memory-driven attentional capture? The effects of memory type, display type, and search type. Journal of Experimental Psychology: Human Perception \& Performance, 35, 1275-1291.

Soto, D., Heinke, D., Humphreys, G. W., \& Blanco, M. J. (2005). Early, involuntary top-down guidance of attention from working memory. Journal of Experimental Psychology: Human Perception \& Performance, 31, 248-261.

Soto, D., \& Humphreys, G. W. (2007). Automatic guidance of visual attention from verbal working memory. Journal of Experimental Psychology: Human Perception \& Performance, 33, 730-737.

Soto, D., \& Humphreys, G. W. (2009). Automatic selection of irrelevant object features through working memory: Evidence for topdown attentional capture. Experimental Psychology, 56, 165-172.
Soto, D., Humphreys, G. W., \& Rotshtein, P. (2007). Dissociating the neural mechanisms of memory-based guidance of visual selection. Proceedings of the National Academy of Sciences, 104, 1718617191.

Theeuwes, J. (1992). Perceptual selectivity for color and form. Perception \& Psychophysics, 51, 599-606.

Theeuwes, J. (2004). Top-down search strategies cannot override attentional capture. Psychonomic Bulletin \& Review, 11, 65-70.

Theeuwes, J., Kramer, A. F., \& Belopolsky, A. V. (2004). Attentional set interacts with perceptual load in visual search. Psychonomic Bulletin \& Review, 11, 697-702.

WoODMAN, G. F., \& LUCK, S. J. (2007). Do the contents of visual working memory automatically influence attentional selection during visual search? Journal of Experimental Psychology: Human Perception \& Performance, 33, 363-377.

Yantis, S., \& Jonides, J. (1990). Abrupt visual onsets and selective attention: Voluntary versus automatic allocation. Journal of Experimental Psychology: Human Perception \& Performance, 16, 121134.

\section{APPENDIX}

\section{Description of Error Rates From the Visual Search, Memory, and No-Go Trials}

We performed a separate ANOVA on the percentage of errors for each type of trial (visual search, memory, and catch). We included attentional window (focused, diffuse) and cue validity (invalid, neutral, valid) as withinsubjects factors, and experiment (visual WM, identification, verbal WM) as a between-subjects factor.

\section{Visual Search Error Rates}

A main effect of attentional window $\left[F(1,89)=4.39, M S_{\mathrm{e}}=21.82, p<.039\right]$ was observed as a result of the error rate being higher when there was a focused attentional window $(2.90 \%)$ than when there was a diffuse attentional window $(2.07 \%)$. The main effect of cue validity was also significant $\left[F(2,178)=10.34, M S_{\mathrm{e}}=\right.$ $15.11, p=.0001]$. Planned comparisons showed a higher percentage of errors in the neutral trials $(2.98 \%)$ than in the valid trials $(1.44 \%)[t(91)<1]$. The search-error data are reported in Table A1.

\section{Memory Error Rates}

For all conditions, the memory data differed from 0 (all $p \mathrm{~s}<.001$ ), indicating that the memory data were not at ceiling. Despite this, there was no significant main effect or interaction in the ANOVA on the memory-error rates (all $F_{\mathrm{S}}<1$ ), and visual inspection indicates that the rates were similar across the conditions. There was no evidence for memory performance benefitting in the valid and invalid cue conditions, as would be expected if participants used the reappearance of the cue to refresh their memory. The memory-error data are reported in Table A1.

\section{No-Go Error Rates}

The main effect of attentional window $\left[F(1,89)=3.06, M S_{\mathrm{e}}=242.35, p<.08\right]$ approached significance, suggesting that there was a higher percentage of errors when there was a diffuse attentional window $(10.94 \%)$ than when there was a focused attentional window (8.63\%). A main effect of cue validity was also observed $\left[F(2,178)=3.87, M S_{\mathrm{e}}=134.36, p<.02\right]$. Planned comparisons showed a higher percentage of errors in the invalid trials $(10.36 \%)$ than the neutral trials $(7.73 \%)[t(91)=2.09, p<.04]$, and a higher percentage of errors in the valid trials $(10.83 \%)$ than in the neutral trials $[t(91)=2.58, p<.01]$. The two-way attentional window $\times$ cue validity interaction $\left[F(2,178)=4.12, M S_{\mathrm{e}}=140.82, p<.02\right]$ and the three-way attentional window $\times$ cue validity $\times$ experiment interaction $\left[F(4,178)=2.65, M S_{\mathrm{e}}=140.82, p<.04\right]$ were also significant.

Separate analyses for each experiment revealed a higher percentage of errors in the valid trials of the diffuse attentional window condition (18.90\%) than in the valid trials of the focused attentional window condition $(10.63 \%)$ in Experiment 1A (visual WM). In Experiment 1B (identification), the percentage of errors was higher in neutral trials with diffuse attention $(13.00 \%)$ than in neutral trials with focused attention $(3.26 \%)[t(30)=$ $3.54, p<.001]$; on valid trials, there was also a trend toward a higher percentage of errors with diffuse attention $(11.07 \%)$ than with focused attention $(7.06 \%)[t(30)=1.78, p<.085]$. No difference was observed between the focused and diffuse attentional conditions in Experiment $1 \mathrm{C}$ (verbal WM; $F<1.0$ ) for the interaction of attentional window $\times$ cue validity). The no-go error data are reported in Table A1. 
APPENDIX (Continued)

Table A1

Error Rates (\%) As a Function of the Size of the Attentional

Window, Cue Validity, and Experiment for Visual Search, Memory, and No-Go Trials

\begin{tabular}{|c|c|c|c|c|c|}
\hline \multirow[b]{3}{*}{ Experiment } & \multirow[b]{3}{*}{$\begin{array}{c}\text { Cue } \\
\text { Validity }\end{array}$} & \multicolumn{4}{|c|}{ Attentional Window } \\
\hline & & \multicolumn{2}{|c|}{ Focused } & \multicolumn{2}{|c|}{ Diffuse } \\
\hline & & $\begin{array}{l}\text { Error } \\
\text { Rate }\end{array}$ & $S D$ & $\begin{array}{l}\text { Error } \\
\text { Rate }\end{array}$ & $S D$ \\
\hline \multicolumn{6}{|c|}{ Search Error Rates } \\
\hline \multirow[t]{3}{*}{ 1A (visual WM) } & Invalid & 5.97 & 8.59 & 3.19 & 4.04 \\
\hline & Neutral & 5.14 & 7.63 & 3.75 & 5.05 \\
\hline & Valid & 2.64 & 6.14 & 0.97 & 2.61 \\
\hline \multirow[t]{3}{*}{ 1B (identification) } & Invalid & 3.29 & 4.99 & 2.46 & 3.74 \\
\hline & Neutral & 3.39 & 6.45 & 3.13 & 4.02 \\
\hline & Valid & 2.54 & 4.92 & 1.29 & 4.00 \\
\hline \multirow[t]{3}{*}{ 1C (verbal WM) } & Invalid & 1.21 & 2.88 & 2.42 & 3.18 \\
\hline & Neutral & 1.34 & 2.49 & 1.07 & 2.14 \\
\hline & Valid & 0.53 & 1.78 & 0.67 & 1.56 \\
\hline \multicolumn{6}{|c|}{ Memory Error Rates } \\
\hline \multirow[t]{3}{*}{ 1A (visual WM) } & Invalid & 8.13 & 7.98 & 6.90 & 6.71 \\
\hline & Neutral & 7.43 & 7.21 & 7.33 & 10.67 \\
\hline & Valid & 7.30 & 7.29 & 6.23 & 8.33 \\
\hline \multirow[t]{3}{*}{ 1C (verbal WM) } & Invalid & 2.93 & 3.72 & 2.65 & 4.06 \\
\hline & Neutral & 3.03 & 3.61 & 2.75 & 4.09 \\
\hline & Valid & 2.91 & 3.34 & 2.18 & 2.84 \\
\hline \multicolumn{6}{|c|}{ Error Rates for No-Go Trials } \\
\hline \multirow[t]{3}{*}{ 1A (visual WM) } & Invalid & 18.96 & 23.02 & 11.20 & 15.41 \\
\hline & Neutral & 10.07 & 12.04 & 8.90 & 12.87 \\
\hline & Valid & 10.63 & 11.95 & 18.90 & 19.40 \\
\hline \multirow[t]{3}{*}{ 1B (identification) } & Invalid & 7.55 & 13.47 & 8.13 & 12.83 \\
\hline & Neutral & 3.26 & 7.99 & 13.00 & 13.38 \\
\hline & Valid & 7.06 & 10.38 & 11.87 & 14.34 \\
\hline \multirow[t]{3}{*}{ 1C (verbal WM) } & Invalid & 8.44 & 14.13 & 10.50 & 13.22 \\
\hline & Neutral & 5.78 & 11.71 & 7.34 & 14.02 \\
\hline & Valid & 7.84 & 13.36 & 11.50 & 17.17 \\
\hline
\end{tabular}

(Manuscript received August 14, 2009;

revision accepted for publication January 18, 2010.) 